\title{
LXXXIX. Positive electricity
}

\section{Sir J.J. Thomson}

To cite this article: Sir J.J. Thomson (1909) LXXXIX. Positive electricity, Philosophical Magazine Series 6, 18:108, 821-845, DOI: 10.1080/14786441208636759

To link to this article: http://dx.doi.org/10.1080/14786441208636759

册 Published online: 21 Apr 2009.

Submit your article to this journal 중

Џll Article views: 9

Q View related articles ๘

Citing articles: 11 View citing articles 
THE

LONDON, EDINBURGH, AND DUBLIN

PHILOSOPHICAL MAGAZINE

AND

JOURNAL OF SCIENCE.

[SIXTH SERIES.]

$D E C E M B E R 1909$.

LXXXIX. Positive Electricity. By Sir J. J. Thomson, Professor of Experimental Physics, Cambridge*.

THE most important questions to be settled as to the 1 nature of positive electricity are:-(1) Does a definite unit of positive electricity exist? (2) If so, what is the size of the unit?

Question (1) may perhaps be made clearer by considering a definite case. Suppose we could get a pure gas, say oxygen or nitrogen, would it be possible to get in such a gas, positively charged particles smaller than the residue left, when a corpuscle is removed from an atom of oxygen or nitrogen?

Again, we know that we can get negative particles of the same kind whether we extract them from oxygen, hydrogen, or nitrogen. Is there anything analogous to this in the case of positive electricity? Can we in short get positively electrified particles of the same kind from different gases?

Our knowledge of charged positive particles (other than a particles) has been derived from two sources : (1) the study of positive particles in systems of positive rays found in vacuum-tubes ; (2) the study of the velocity of the positive ions in ionized gas.

To begin with the first method. The two sets of positive rays which have been most closely studied are, firstly, the Canalstrahlen which are found passing through a perforated

* A paper introducing a discussion on this subject read on August 30, 1909, at the Winnipeg meeting of the British Association. Communicated by the Author.

Phil. Mag. S. 6. Vol. 18. No. 108. Dec. 1909. $3 \mathrm{~K}$ 
cathode, and, secondly, the rays shot out from the cathode in the same direction as the cathode rays but which are deflected much less than those rays by magnetic forces, and which instead of carrying a negative clarge are some of them positively charged while others are electrically neutral.

To take the Canalstrahlen first. These can be studied by an apparatus like that represented by fig. 1. It consists of a

Fig. 1.

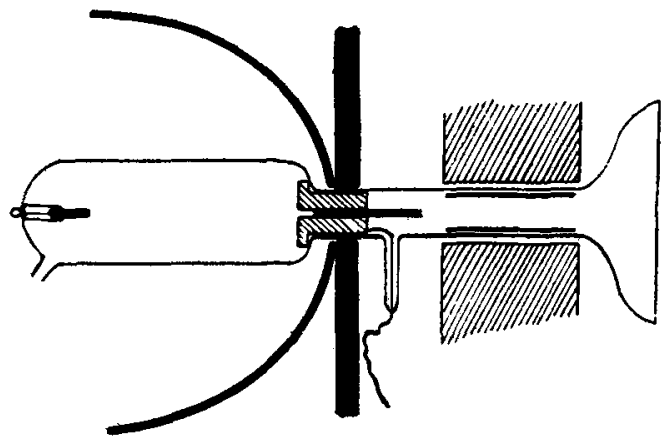

vacuum-tube with a perforated cathode through which a beam of Canalstrahlen is streaming. The rays strike against a willemite screen at the end of the discharge-tube and produce phosphorescence when they reach the screen. In their path to the screen the rays pass between the poles of an electromagnet which deflects them in a vertical direction and simultaneously between two parallel metal plates connected with the terminals of a large number of storage-cells; the electrostatic field deflects the spot horizontally. The magnetic deflexion is equal to $\mathrm{B} e / m v$, where $e, m, v$ are respectively the charge, mass, and velocity of the particles, and $B$ a constant depending upon the strength of the magnetic field and the distance of the screen from the cathode. The electrostatic deflexion is equal to $\mathrm{A} e / m v^{2}$, where $\mathrm{A}$ depends on the strength of the electric field and the size of the apparatus. When both these fields are in activity, the appearance presented by the willemite screen when the rays strike against it is, when the pressure is not very low, somewhat like fig. 2. The small spot which marks the place of incidence of the pencil when the magnetic and electrostatic fields are not on, is drawn out into a ribbon the edges of which are often very approximately straight. In some cases the original position of the spot, even when the magnet is on, is more brightly 
illuminated than any other part of the ribbon ; in others the illumination is fairly uniform throughout.

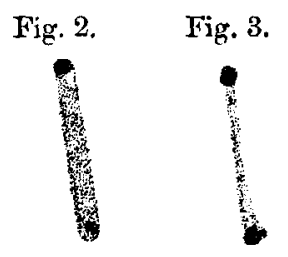

On the other side of the undeflected position there is a luminous tail, formed, as the direction of the deflexions shows, of negatively electritied particles. The brightness of this in comparison with the positive part changes very considerably : in some cases it is too faint to be ensily perceived, in others it is sometimes almost as bright as the positive part of the ribbon ; in general, however, it is much feebler and there is distinct discontinuity in the brightness of the light in the tail and in the other portions.

If $y$ is the vertical, $x$ the horizontal deflexion of the rays,

$$
\begin{gathered}
v \propto y / x \\
e / m \propto y^{2} / x .
\end{gathered}
$$

Thus when the ribbon is straight, the values of $y / x$ and therefore the velocity of the particles are approximately constant, while $y^{2} / x$ ranges from zero up to a maximum value at the end of the strip. This maximum value, in all the cases I have tried, gives a value of $e / m$ equal to $10^{4}$.

When the pressure of the gas is reduced, the luminosity of the band concentrates into a spot or spots and presents the appearance shown in fig. 3. There is a bright spot at the end and a faint band which at low pressures becomes almost invisible connecting this spot with the position of the undeflected spot; at this place there is not infrequently a considerable amount of luminosity even when the magnet is on. This luminosity is often indistinct and sometimes vanishes. When it is present it indicates the presence of a considerable number of rays which are not deflected in passing through the magnetic and electric fields, and which are therefore not charged when they pass through these fields. Neglecting for a moment the faint luminosity connecting the spots, the bundle of rays in this case consists of a mixture of positively electrified particles and electrically neutral systems, uncharged rays we may call them; these uncharged rays, however, in their course through the gas may gradually get broken up $3 \mathrm{~K} 2$ 
and become positively charged rays. The breaking up of these uncharged rays may be shown and the rate at which they break up measured by the following method. A tube (fig. 4) in which the distance between the perforated cathode

Fig. 4.

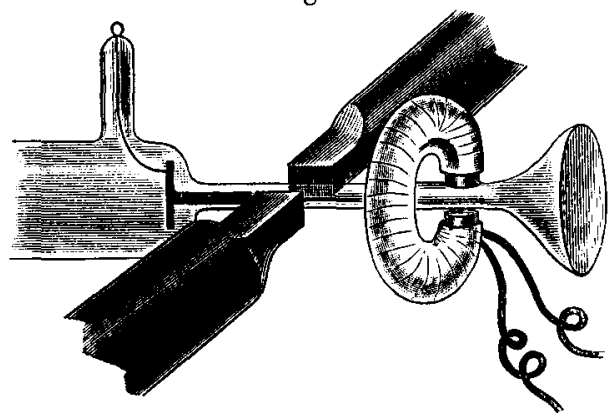

and the willemite screen was considerably greater than in the tube fig. 1, was made, and two electromagnets arranged, one close to the cathode, and the other much nearer the screen. The magnets were arranged so that the magnetic force in the first was horizontal, and the deflexion of the spot due to the field vertical, while in the second magnet the magnetic force was vertical and the deflexion horizontal. The deflexions due to each magnet were thus separated and could be measured independently.

The effects observed when the magnets were applied in succession and then simultaneously are interesting; and a typical case is represented in figs. 5 \& 6 . Fig. 5 represents the appearance of the screen when only the electromagnet next the cathode is in action; fig. 6 the appearance when both magnets are on.

In fig. 6 the undeflected spot is much fainter than in fig. 5; part of it is deflected to another spot $a^{\prime}$, while the spot $b$ is deflected to $b^{\prime} . \quad b^{\prime}$ is vertically under $a^{\prime}$, so that the deflexion produced by the second electromagnet on the rays which were uncharged when they went through the first electromagnet is the same as that produced by the second magnet on the rays which were charged when they went through the first one. It is evident that the latter have for the most part kept their charge from the cathode right through the second magnet; the formation of the spot $a^{\prime}$ shows that some of the neutral rays have in their journey from the first magnet to the second split up into a positive and negative part (the negative part being deflected far out of the field and not 
reaching the screen), while the positive has been affected by the magnet to just the same extent as those which were positively electrified in the field of the first magnet; this shows that the rays which break up in the journey move with the same velocity and possess the same charge as those which break up at an earlier stage.

Fig. 5.

Fig. 6.

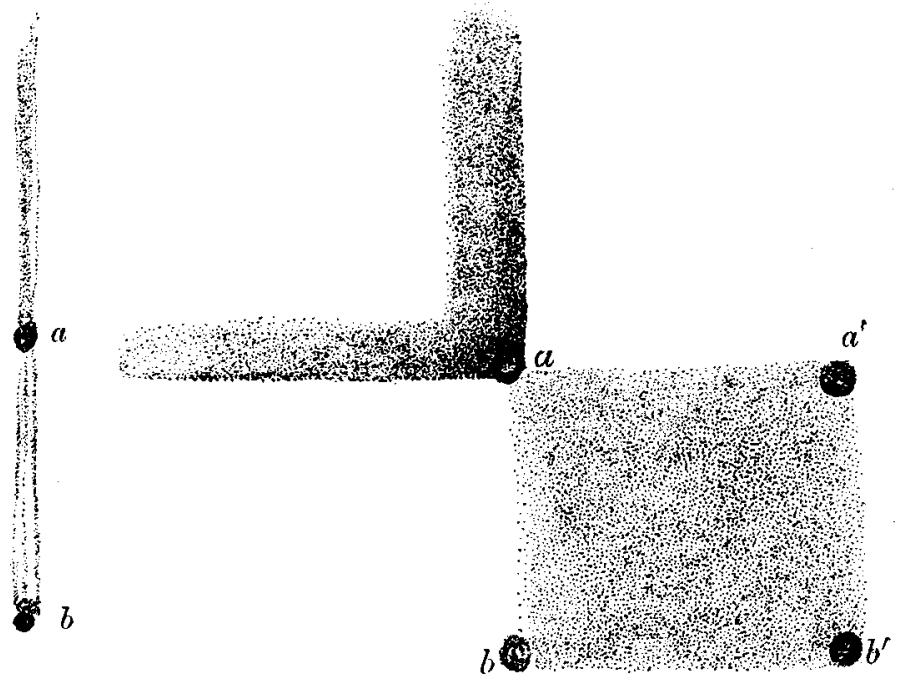

The relative brightness of the spots $a$ and $b$ varies a good deal with the pressure. I have found that when the pressure is reduced the spot $a$ gets fainter than $b$, though at higher pressures it might have been brighter. This is what we should expect, since at the lower pressure the neutral doublets make fewer collisions in their course between the two magnets, and so fewer of these are broken up into ions. Again, as the pressure increases, the spots become less wolldefined, there is more luminosity between the original and the displaced positions of the spots ; for example, there may be considerable luminosity between the position of $b$ when the vertical magnetic field is off and $b^{\prime}$, the luminosity stretching right up to $b^{\prime}$.

This shows that at this pressure some of the particles which were positively charged when they were passing through the first electromagnet have got neutralized before reaching the second and so are not deflected by it. The breaking up and re-formation of the doublets become more 
frequent as the pressure increases, and more and more particles are positively charged for only part of the time they are in the magnetic field. Since they are only deflected when charged, the deflexion will vary with the time they have been uncharged while passing through the magnetic field; and if this time varies continuously the spot will be drawn out by the magnet into a continuous band such as is represented in fig. 2, and which is always observed when the pressure is not too low.

An important point as to the determination of $\mathrm{e} / \mathrm{m}$ occurs in connexion with this effect : the values of $\mathrm{e} / \mathrm{m}$ got from the magnetic and electric deflexions are the average values of $e / m$ whilst the system is in the magnetic and electric fields.

Since some of the systems are at times electrically neutral, the average value of $\mathrm{e} / \mathrm{m}$ cannot be greater than the true value, and it may be considerably less. It may be asked, have we any security when we deduce the value of $\mathrm{e} / \mathrm{m}$ for the positive ions from the measurement of the tip of a drawn out band such as that in fig. 2, that the particle under consideration has been charged the whole time it was passing tnrough the field ; if this is not the case, the true value of $e / m$ may be much greater than the value calculated.

This consideration does not apply to the case when the bright spot is deflected as a spot and not as a band; but a great many determinations of $e / m$ have been made when the pressure was too high to give spots, and the ends of the bands have been used for this purpose.

I have made some experiments to see if in my measurements any errors have been made from this cause. The principle of these experiments was as follows. If the particle at the tip of the beam has not been charged the whole time, $i . e$. if it has united with a corpuscle before leaving the magnetic field, then the value of $e / m$ determined by this method will increase as the length of the path travelled in the magnetic field is reduced until it is comparable with the length of the path described by the particle whilst it is positively charged; and when the magnetic field is so short that the particle can retain its charge for the whole of the distance between the poles of the magnet, the value of $e / m$ given by this method would rise to a maximum.

Now take two magnetic fields, one short but strong, the other long but weak, and adjust the strengths of the fields so that if the positive particle retained its charge the whole of the time it was in either field, the deflexion of the particle would be the same in both cases. If the particles whose 
deflexion we are measuring become neutralized in passing through the longer field, the deflexion produced by the short field will be greater than that by the long, provided the length of path of the particle when uncharged is comparable with the length of the shorter field.

For this experiment, chisel-shaped pole-pieces whose ends were rectangular were used, the longer side of the rectangle was $4.5 \mathrm{~cm}$., the shorter side $\cdot 7 \mathrm{~cm}$. The distance between the pole-pieces was $6 \mathrm{~mm}$. The poles were arranged (1) so that the longer side of the rectangle was parallel to the path of the positive particle, so that this was exposed to a magnetic field for about 5 cms. of patb, and (2) with the shorter side parallel to the path so that the particle was exposed to the magnetic field for less than a centimetre of path. The currents through the electromagnets were adjusted so that the deflexion would be the same for a particle which retained its charge the whole of the time it remained in either field. It was found under these circumstances that when the pressure was too high to give well-defined spots the deviations of the tips of the luminous bands into which the phosphorescence was drawn out were the same in both cases; showing that the maximum value of $e / m$ got by this method is the ratio of the charge on the particle to its mass, and not the average extending over a time during part of which it is uncharged.

Though the maximum deflexion is the same with the two fields, there are minor differences in the appearance of the phosphorescent patch ; with the short field the portion of the band which is not deflected at all is noticeably brighter than in the longer field. This is what we should expect if the rays contain large quantities of neutral doublets which break up into positively and negatively electrified parts as they pass through the tube and collide against the molecules of the residual gas.

The behaviour of the portion of the luminosity due to negatively charged portions of the Canalstrablen is interesting and throws light on their origin; the determination of $e / m$ for these show's that they are not corpuscles but that their mass is much the same as that of the positive particles, they are in fact neutral doublets which have acquired a negative charge.

'I'be brightness of the negative part of the phosphorescence depends very much on the intensity of the magnetic field; as the intensity of the field increases these negative rays at a certain stage increase so quickly in brightness as to give the impression that they suddenly spring into existence. Again, 
with a constant magnetic field the negative rays are much brighter when the magnetic force is at some distance away from the cathode and towards the screen than when it is applied nearer to the cathode.

The action of the magnetic field in increasing the number of these negatively electrified particles is easily understood, for in a strong field the negative corpuscles can only move along the lines of force and thus get concentrated into a cloud ; an unelectrified doublet passing through this would be very liable to get electrified.

There is in general an abrupt falling off in the intensity of the luminosity under electric and magnetic forces as we pass from the position of the spot corresponding to uncharged rays into the position corresponding to negatively charged rays. This shows that the uncharged rays are not rays which start by being positively electrified, then take up a corpuscle and get neutralized, then another corpuscle and become negatively electrified, then lose a corpuscle and so on, repeating this process so that on the average the charge is as often positive as negative ; for if this were the case, the number of these would be very nearly the same as that of those which had slightly overshot the mark and become on the average just a little more negative than positive, and these would be found amongst the negative rays, and the transition from the one to the other would be gradual.

Everything points, I think, to the conclusion that even at the start from the cathode the Canalstrahlen include a large number of neutral doublets, if indeed they do not wholly consist of them.

These neutral doublets are found not only in Canalstrahlen, the rays at the back of the cathode which have passed through it, they are also found in front of the cathode travelling away from it, mixed up with rays which carry a positive charge of electricity and which are thus travelling against the electric field.

The values of $e / m$ for these positively charged rays in front of the cathode are, as I have shown (see Phil. Mag. Oct. 1908), the same as for the Canalstrahlen; it is convenient to call the positively charged rays which travel away from the cathode in the same direction as the cathode rays, "Retrograde rays." The presence of neutral doublets is even more marked among the retrograde rays than among the Canalstrablen.

These neutral doublets are very interesting, as they form an intermediate stage between the ion and the neutral molecule. They are found not only in these high tension 
discharges, but also when the electric field is much less intense than when the discharge is produced by the inductioncoil. This can be shown with an apparatus like that shown in fig. 7, where $W$ is a hot Wehnelt cathode, connected up

Fig. 7.

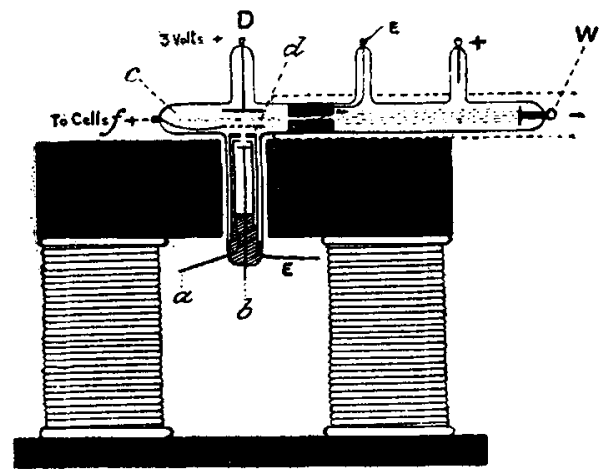

with a battery of small storage-cells, cansing it to emit slow cathode rays which ionize the gas in the tube $\mathrm{C}, a$ and $b$ are two carefully insulated Faraday cylinders; there is a very small hole, in some cases less than $\frac{1}{2} \mathrm{~mm}$. in diameter, in the plate $d$ which separates the Faraday cylinders from the ionized gas ; through this hole the ions produced in $\mathrm{C}$ can diffuse and charge up the Faraday cylinders.

We can, however, by suitable means prevent the ions passing through the hole: the negative ones which are mainly corpuscles, and easily deflected by a magnet, are prevented by applying a strong magnetic field which bends them back before they reach the cylinder ; the positive ions, which are not easily deflected by a magnet, can be stopped by a strong electric field between the wire gauze $f$ and the top of the cylinder $d$, the gauze being negative to the cylinder. When the ions are stopped by these means we find that though no perceptible electric charge reaches either cylinder, the gas between them is a conductor of electricity, and if either cylinder is charged up the other wili slowly acquire the same potential, whether that potential be positive or negative.

That the conductivity of the gas is not due to ultra-violet light coming from the luminous discharge in the upper tube is shown by the fact that it is destroyed by putting a thin quartz plate over the hole in $d$; and since it is also destroyed when a piece of the thinnest aluminium foil obtainable is placed 
over the hole, it cannot be due to any ordinary form of Röntgen radiation.

The conductivity can easily be explained if we suppose that there are neutral doublets in the ionized gas in the dischargetube, and that since these are not deflected by either electric or magnetic forces they can pass through the hole though the ions are stopped. Then by collision with the molecules of the gas in the Faraday cylinder, they break up into ions and thus make the gas a conductor.

The velocity of the neutral doublets in the discharge produced by an induction-coil is approximately the same as that of the positively charged particles which accompany them. This follows from the fact that the deflexion of the spot $b$ (fig. 6) is the same as that of spot $a$, though the former spot is produced by positively charged particles which have been detached from the neutral doublets after passing the first magnetic field, and the second by positively electrified particles which were free before the first field was reached.

The doublets seem to travel in directions approximately normal to the surface of the cathode. Experiments described in my paper on "Positive Rays" (Phil. Mag. Oct. 1908) show that the retrograde rays travel in this direction, and it is well known that Canalstrahlen are not obtained unless the aperture in the cathode is approximately normal to the face of the cathode facing the anode. The direction of the doublet is in short approximately that of the lines of force close to the cathode; they may move in either direction along the lines.

The doublets seem to start from beyond the dark space next the cathode, for if a vertical obstacle such as a thin glass rod or a metal wire be placed inside the discharge-tube in such a position that its projection on the plane of the cathode passes across the hole in the cathode through which the Canalstrahlen pass, then when the pressure is so low that the dark space extends beyond the obstacle, the dark shadow of the obstacle is apparent on the willemite screen, stretching across the phosphorescent patch even when this is spread out by the electric and magnetic fields. If the pressure is increased so that the obstacle is outside the dark space and in the negative glow, no shadow can be perceived.

In this form of the experiment the shadows cast by an obstacle are much more easily perceived and much more definite than in the original form in which it was tried by Schuster and Wehnelt. I have got quite sharp shadows with a fine wire placed several centimetres away from the cathode.

Some very interesting questions arise in connexion with 
these doublets. Is the doublet the thing set free from the atom when it is ionized? and are the ions produced by the splitting up of the doublets, or are the ions first produced and the doublet produced by a combination of the positive and negative ions?

There are arguments to be urged in favour of either view. The fact that the direction of projection of these doublets is parallel to the direction of the lines of force at the cathode, points to the projection being due to electrical forces.

We can easily understand how these might set the doublets in rapid motion.

Thus doublets which accompany the retrograde rays may have had a corpuscle attached to them when they were in the neighbourhood of the cathode, and before losing this corpuscle have been projected away from the cathode in the same direction as the cathode rays; thus these doublets acquire their velocity when they are doublets. The donblets which accompany the Canalstrahlen and which move in the opposite direction to the preceding, may be supposed to have acquired their velocity when they were in a dissociated state; $i$. e., we may suppose that a positive ion in front of the cathode acquires in its fall to the cathode a high velocity, and then after passing through the cathode unites with a corpuscle and becomes a doublet retaining the velocity acquired by its positive constituent.

We have already had examples of the formation of doublets in the Canalstrahlen group. Thus, for example, in the case represented in fig. 6 , we saw that when the pressure was not very low, some of the positive ions which had retained their charge whilst passing through the first magnetic field, and which form the spot $b$, had lost their charge, i.e. had become doublets before entering the second field and were not deflected by it, so that instead of the spot being entirely removed when the second magnetic field was put on, some of it was left behind.

Though we can hardly doubt that there must be positively charged particles passing through the hole in the cathode which owe their velocity to this cause, yet, as the experiments I shall now proceed to describe show, Canalstrahlen such as those used when their electric and magnetic deflexions are measured in the way I have described and which travel perhaps $15 \mathrm{cms}$. before they reach the screen, seem to owe their velocity to a different cause, for I have found that the velocity of these is but slightly affected by the potentialdifference between the electrodes of the discharge-tube or the pressure of the gas. 
I have made many determinations of the velocity of Canalstrahlen at different pressures using tubes of very different shapes, and the results were so nearly constant in spite of the wide variations in the conditions, that $I$ was led to suspect that the velocity of the positive rays was not influenced to any great extent by the strength of the electric field in the tube. To test this matter in as direct a way as possible I used the following method.

Fig. 8.

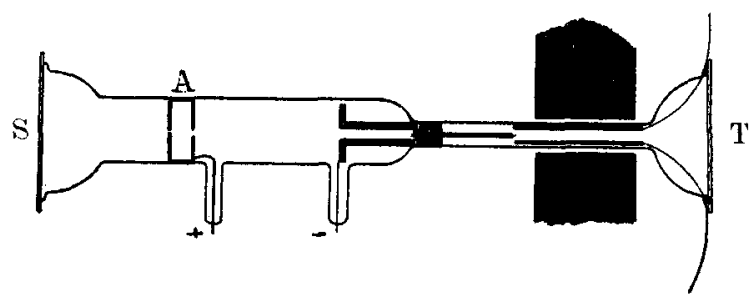

This discharge was produced by a large induction-coil ; the discharge-tube, fig. 8, had a perforated cathode faced with calcium, so that the discharge would pass at very low pressures without sparking through the glass walls of the tube and breaking the apparatus. The anode $A$ was an aluminium cylinder perforated with a very small hole. When the discharge was passing through the tube a pencil of cathode rays passed through the hole and produced a small well-defined spot on the fluorescent willemite screen $\mathrm{S}$, placed at the anode end of the tube.

During their path up to the screen after leaving the anode they could be exposed to a known magnetic field produced by an electromagnet, and the distance through which the spot moved when the magnet was put on enabled us to calculate the velocity of the cathode rays.

The Canalstrahlen after passing through the aperture in the cathode pass along a metal tube of very fine bore (one of the perforated needles used for hypodermic injections) and emerge between two parallel vertical metallic plates. $(4 \mathrm{~mm}$. apart in one set of experiments, $3 \mathrm{~mm}$. in another) which can be connected with the terminals of a battery of small storage-cells; the rays are thus exposed to a strong. electric field and are deflected horizontally.

Two poles (about the same length as these plates) of a powerful electromagnet of the $\mathrm{Du}$ Bois pattern, are placed on either side of the tube, and the horizontal magnetic field due to this magnet deflects the rays in a vertical direction. The deflexion of the rays is measured by the deflexion of the 
phosphorescent patch they produre on the willemite screen $\mathrm{T}$; from the magnetic and electric deflexions the values of $e / m$ and $v$ can be deduced in the usual way. Observations were made simultaneously of the deflexions of the spots on the screens $\mathrm{S}$ and $\mathrm{T}$ when the pressure was varied from the largest value at which both rays produced phosphorescence on the screen to a very high vacuum. The deflexion of the cathode rays under a constant magnetic field was $8 \mathrm{~mm}$. at the highest pressure and $2 \mathrm{~mm}$. at the lowest; thus the velocity of the cathode rays was 4 times greater at the lower pressure than at the higher, but in spite of this large change in the catbode rays, there was no appreciable change in the deflexion of the Canalstrahlen over the whole range of pressure.

The results of two sets of experiments with different tubes are shown in the following table.

By equivalent spark-gap is meant the distance between two large electrodes placed in parallel with the tube and adjusted so that the sparks passed about equally readily through the tube or across the air-gap.

\begin{tabular}{|c|c|c|c|}
\hline Deflexion of Canalstrahlen. & $\begin{array}{c}\text { Deflexion of } \\
\text { Dathode Rays. }\end{array}$ & $\begin{array}{c}\text { Equivalent } \\
\text { Spark-Gap. }\end{array}$ \\
\hline Magnetic. & Electrostatic. & mm. & $\mathrm{cm}$. \\
\hline $\mathrm{mm}$. & $\mathrm{mm}$. & 6 & $\cdot 5$ \\
6 & 6 & $4 \cdot 5$ & \\
6 & 6 & $2 \cdot 5$ & $3 \cdot 8$ \\
5.5 & 6.5 & 2 & \\
6.5 & 6 & $\mathrm{~mm}$. & \\
\hline $\mathrm{mm}$. & $\mathrm{mm}$. & 6.5 & \\
6 & 4 & 5 & \\
6 & 4.5 & 2 & \\
\hline 6 & 4 & 35 & \\
\hline
\end{tabular}

The numbers in one horizontal line were observed simultaneously, one observer reading the deflexion of the cathode rays, another those of the Canalstrahlen. At the very highest pressures at which Canalstrahlen appear their deflexions seem greater than those at lower pressures, though the difference is on quite another scale to that of the cathode 
rays. The phosphorescence at these pressures is very diffuse and faint and the rays are evidently much scattered in their journey to the screen. I think the increased deflexion is due more to this and their loss of velocity during the journey, than to any considerable falling off in their initial velocity of projection.

Though the equivalent spark-length has changed in the ratio of nearly 8:1 and the deflexion of the cathode rays as $3: 1$, there has been hardly any change in the deflexions of the Canalstrahlen. This makes it very improbable that they owe any considerable part of their velocity to the action of the electric field upon them whilst they approach the cathode.

We must remember that the bundle of Canalstrahlen when it passes through the hole is a mixture of rays of different kinds. With such an apparatus as that just described the only rays investigated are those which can traverse the very considerable distance between the cathode and the screen and yet retain their power of producing phosphorescence. Coming through the hole there may be, in fact in certain cases we know there are, positive particles which have been set in motion by the electric field, with velocities depending on those of the molecules of the gas in the tube.

These, in the experiment I am describing, have disappeared before travelling as far as the screen, their " range," to use an expression familiar to those who study the $\alpha$ particles, is less than the distance between the screen and cathode. The existence of this range in the case of the $\alpha$ particles may be explained by supposing that when the velocity of the particle falls below a certain value, the particle is no longer able to escape from the negative corpuscles which it has to pass through on its travels. The same argument will apply to Canalstrahlen; if they move with less than a certain speed they may not be able to escape from corpuscles near which they pass. Since the $\alpha$ particle has twice the charge of a particle in the Canalstrahlen, the velocity, other circumstances being the same, required for the escape of an a particle will be greater than for one in the Canalstrahlen. The velocity of the latter, in round numbers $2 \times 10^{8} \mathrm{~cm}$. per sec., is about that required for a particle of unit charge and $10^{-8} \mathrm{~cm}$. in radius to escape from a corpuscle.

In the preceding experiments the velocity of the cathode rays at the lowest pressure was $6 \times 10^{9} \mathrm{~cm}$./sec., taking $e / m=1.7 \times 10^{7}$. The velocity of the Canalstrahlen particle is thus considerably less than that of a cathode ray particle; the energy of a cathode particle is, however, less than that of a particle in the Canalstrablen. 
Experiments were also made with the retrograde rays, and it was found that the velocity of these was also practically independent of the strength of the field and very nearly the same as that of the Canalstrahlen.

The small range of velocities in the Canalstrahlen is explicable if we suppose that the rays we are measuring exist originally as neutral doublets which are formed by the combination of a positive unit and a negative corpuscle; these doublets moving towards or away from the cathode, the direction of motion being approximately at right angles to the cathode. These doublets could not have more than a certain amount of energy ; for if the relative velocity of the carriers of the positive and negative charges when uncombined exceeded a certain value, the charges would fly past each other without entering into combination and they would remain dissociated : it is only when the kinetic energy of the two falls below a certain value that combination will take place; thus there will be a superior limit to the energy of the doublets. The doublets which we investigate when we measure the velocity of the cathode rays are those which dissociate again into positive and negative charges. Now, as the kinetic energy of the doublet decreases it gets more and more stable and less likely to dissociate, and when it ceases to dissociate we cannot measure the velocity. Thus doublets are not formed possessing energy greater than a certain value; on the other hand, they are not split up if the energy is less than a certain value. 'I'hus the doublets whose velocity we can determine will have velocities between definite limits which are independent of the strength of the electric tield. The case is analogous to that of a gas which dissociates as the temperature is raised : there is a certain range of temperature in which there is appreciable, but not complete dissociation; beyond the upper limit of this range of temperature the undissociated molecules are too few to be detected; beneath the lower limit the products of dissociation are not numerous enough to be appreciable.

The properties both of the Canalstrahlen and the retrograde rays could also be explained on the view that neutral doublets moving with high velocities are given out by the molecules of the gas in the discharge-tube, that these break up into the Canalstrahlen and the retrograde rays; these doublets are supposed to be of the same character from whatever kind of gas they may originate. We should, however, expect that these doublets shot out from the molecules by explosions would be found moving in other directions than just along the lines of force in the tube, which seems to 
be the only direction in which an appreciable number of Canalstrablen or retrograde rays move.

If doublets form an intermediate stage between the ions and the atoms, the doublets being the systems formed from the molecules of the gas by the ionizing agent, say Röntgen rays, then the number of doublets liberated may be enormously greater than the number of ions set free, so that this number may give no indication of the percentage of molecules affected by the rays.

'Thus, suppose $q$ doublets are liberated per second per cubic centimetre in a gas at a pressure $p$, let $\mathrm{D}$ be the number of doublets per cubic centimetre, let $\beta p \mathrm{D}$ of these reunite with the molecules per sec., and $\gamma p \mathrm{D}$ split up into ions, let $m$ be the number of positive or negative ions per cc., a the coefficient of recombination; then we have

$$
\begin{aligned}
& \frac{d \mathrm{D}}{d t}=q-\beta \mathrm{D} p-\gamma \mathrm{D} p, \\
& \frac{d m}{d t}=\gamma \mathrm{D} p-\alpha m^{2} .
\end{aligned}
$$

When things are in a steady state

$$
m=\frac{1}{\sqrt{\alpha}} \sqrt{\frac{\gamma}{\beta+\gamma}} q .
$$

The saturation current is proportional to the value of $d m / d t$ when $m$ is zero ; hence, when things have settled into a steady state, the saturation current is proportional to $\frac{\gamma}{\beta+\gamma} q$.

Thus, if $\gamma$ is very small compared with $\beta$, the number of ions taken from the gas per second may be very small compared with the number of molecules affected by the rays. This point is an important one in connexion with the structure of the Röntgen rays, the small percentage of the molecules decomposed has always been difficult to understand.

If we suppose that the ions are the first product of the Röntgen rays on the molecules, this difficulty disappears on the assumption that there is a stage intermediate between the molecules and the ions.

The values of $e / m$ for the Canalstrahlen and for the retrograde rays are the samo when the pressure is low whatever be the gas in the tube. In a former paper I described experiments which showed that if a vessel was exhausted until the pressure was so low that the discharge would not pass, and small quantities of hydrogen, helium, air, oxygen, 
carbonic acid, or argon, were introduced so as to raise the pressure sufficiently to produce the discharge, the values of $e / m$ and the velocity of the particles were the same for all gases. Since then I have repeated the experiments and bave tried in addition to the gases I have mentioned, sulphur dioxide, methyl iodide, carbon tetrachloride, and also the vapour of a radioactive substance $\mathrm{UrCl}_{4}$. With all these gases the values of $e / m$ for the Canalstrahlen were the same and equal to $10^{4}$. This seems strong evidence that there is a definite unit of positive electricity as well as of negative, the carrier of the positive unit being much larger than the negative. This view seems to mo to be very much strengthened by some results recently obtained by $\mathrm{Mr}$. Wellisch at the Cavendish Laboratory. The experiments were on the mobility of the positive and negative ions in mixtures of different gases. If we take the view that the positive ion is but the residue left after a corpuscle has been abstracted from the molecule, then it would seem probable that in the case of positive ions there would, in a mixture of two gases $\mathrm{A}$ and $\mathrm{B}$, be two different types of positive ions moving with different velocities. In one type the positive ion would be the molecules of A minus a corpuscle, or an aggregate formed round it; in the other the nucleus would be the molecule B. Mr. Wellisch found, however, as M. Blane had previously found for a mixture of air and carbonic acid, that in the mixture all the positive ions moved with the same velocity.

We might, however, without assuming an independent unit of positive electricity, explain this result by supposing that the ions were aggregates continually breaking up and reforming, and that the velocity measured is the velocity of the average aggregate. This explanation is, however, refuted by some of the cases examined by Mr. Wellisch. The ionization under Röntgen rays of methyl iodide, mercury methyl, or carbon tetrachloride, is so vastly greater than that of hydrogen, that in mixtures of these gases with hydrogen, even when there is only one or two per cent. of these gases in the mixture, practically the whole of the ionization is due to the methyl iodide. Wellisch found that in these mixtures the velocity of the positive ion is nearly the same as in pure hydrogen, although practically all the ions came from the heavy gases. If the positive ion was merely the residue left when the corpuscle was taken out, the positive ion would be at least as large as a molecule of methyl iodide. We can show, however, that the velocity with which the charged molecule would move through the Phil. Mag. S. 6. Vol. 18. No. 108. Dec. 1909. $3 \mathrm{~L}$ 
mixture, would be less than the observed velocity of the positive ion, which was about that of a charged hydrogen atom. It is clear, then, that the positive ion is not the molecule of methyl iodide. Thus the ionization of the methyl iodide has produced positive ions which are not molecules of methyl iodide. It might perhaps be urged that the molecules of methyl iodide which were positively charged to begin with, have, by the electric force they exert, pulled a corpuscle out of the neighbouring hydrogen molecule, and that this corpuscle neutralizes the methyl iodide molecule and leaves the hydrogen molecule, from which it has escaped, with the positive charge. There are several objections to this view of which I will only mention one. The result of this process is, by the electrical forces between the molecules of methyl iodide and hydrogen, to transfer the charge from the larger system (the methyl iodide molecule) to the smaller (the hydrogen molecule).

Since the potential energy of a given charge is greater when the charge is on a small system than when it is on a large one, the change under consideration would involve an increase in the potential energy, and would therefore not be brought about by the action of the electrical forces.

The results obtained by Wellisch are exactly what we should expect if there were a definite positive unit of electricity which could, like the corpuscle, be detached from the molecule of the gas. I have elsewhere suggested that the process of ionization consists in the detachment of a neutral doublet from the molecule, the doublet consisting of a positive unit and a corpuscle, and that this breaks up into its components, which subsequently attach themselves to the molecules by which they are surrounded.

\section{Slowly moving Positive Rays and the Size of the Carriers.}

The slowly moving rays were studied in the following way :-The gas at a very low pressure was ionized by the cathode rays emitted from a Webnelt cathode W (fig. 7,p. 829). By means of a feeble electric field between the plate D, called the collector, and a piece of wire gauze $f$, the positive ions were then driven through this gauze, which was distant about $.5 \mathrm{~mm}$. from the top of the plate $d$.

Between this gauze and the top of the plate was the potential relied upon to give the positive ions their velocity.

The difference of potential between the collector plate and the gauze was 10 volts, that between the gauze and $d$ varied from 40 to 200 volts. There was a very small hole in the 
plate $d$, and the positive ions, after passing through the hole, travelled through the strong magnetic field produced by a $\mathrm{Du}$ Bois magnet. The magnetic deflexion of the rays was measured by the ratio between the charges received by the disk $b$ and the cylinder $a$ : these were connected with Wilson tilted electroscopes, and the ratio of the charges measured in the way described in my paper on the Positive Rays (Phil Mag. Oct. 1908).

If $V$ is the fall of potential between the gauze and the top of the plate,

$$
e V=\frac{1}{2} m v^{2}
$$

while the magnetic deflexion gave the value of $m v / e$.

Thus from these results $m / e$ and $v$ can be determined.

We find that for these slowly moving positive rays the velocity depends upon the strength of the electric field.

The point that $I$ wish to dweIl upon here is, that in some cases the method seemed to give values of $e / m$ for the positive ions much greater than $10^{ \pm}$. I think these high values are not genuine, but I will first describe the effects which seem to indicate them. We may get, perhaps, the clearest idea of these by describing what happens when the arrangement is altered so as to send negative ions and not positive through the same hole in the top plate.

In this case the corpuscles and negative ions pass through the plate.

If we compare the charges on the disk and the cylinder when there is no magnetic field on, we find that in general there is more negative charge on the cylinder than on the disk, and the effect of a small magnetic field, say 200 units, is greatly to diminish the proportion of the negative charge on the cylinder to that on the disk: when the magnetic force is greatly increased this proportion again increases, due to the deflexion by the magnel of the heavy negative ions from the disk to the cylinder.

It will be noticed that the effect of putting on the small magnetic field is to diminish the scattering. This is what we shonld expect if these were negative corpuscles getting through the hole when there was no magnetic field. If these rays were rather diffuse they would reach the sides of the cylinder.

Now when a magnetic field of say 100 units is put on, it twists up the path of the particles into very small circles, so that they are prevented by it from even getting as far as either of the Faraday cylinders.

The magnetic field is not sufficient to affect appreciably $3 \mathrm{~L} 2$ 
the heavy negative ions which move on to the disk, and are not deflected until the magnetic force is strong enough to deflect ions whose mass is comparable with that of atoms.

I have occasionally observed a similar effect when the positive instead of the negative ions were sent through the oylinder.

This effect has only been observed when the potentialdifference between the gauze and the top of the plate exceeded about 120 volts, and then only occasionally; still, on several occasions it was found that the amount of positive electricity going to the cylinder when there there was no magnetic field on, was a larger multiple of that going to the disk than when the magnetic field was increased to about 100 units : this effect is similar to that observed with negative electricity and which could be explained, as we saw, by supposing that there were particles of exceedingly small mass along with the others. Are we to conclude that there are similar small positive particles, particles whose mass is very small compared with that of the atom? I do not think that the effect I am describing is due to this. In the first place, while the effect is invariably observed with negative electricity, it is quite exceptional with positive.

I had worked with the apparatus described for months, making observations almost daily, before I found an example of it. I think that it arises from the presence among the stream of positive ions which pass through the bole in the plate, of some ions which are moving with much smaller velocity than that which they would acquire by a fall through a potential-difference equal to that between the gauze and the plate.

We have seen that these positive ions are continually getting neutralized in their journey through the gas, and that some of them are hardly deflected at all, that is, do not acquire an appreciable velocity whilst they are passing through the electric field.

Thus we might expect that some of the ions would be neutralized for part of their journey between the gauze and the plate, and would thus only acquire a small fraction of the velocity due to a fall through the whole potential-difference. between the plates.

These slowly moving ions would be easily deflected, and a comparatively weak magnetic field will be sufficient to prevent them entering the Faraday cylinders. If the effect I am describing is due to the slowly moving ions, it ought 
to disappear if the Faraday cylinders are insulated and maintained at a potential a few volts above the plate $A$. The ions, which have acquired an energy due to the fall through the whole potential-difference of say 160 volts, will be but little affected by having to go against the difference of say 20 volts, but those ions which have acquired only a fraction of this velocity may be wholly stopped by it. On raising the potential of the cylinders to about 20 volts, I found that the effect in nearly every case disappears.

There were, however, one or two cases in which it still remained; if the slow moving particles remain neutralized for part of their journey between the plate and the cylinder, they could not be much affected by the field, and the positive particles might still force their way in.

As the question of the existence of small positive ions is a very vital one, I have made some other investigations with other cases of easily deflected positive ions with the object of seeing if the deflexions were in these cases also due to the smallness of the velocity rather than to that of the mass.

The appearance of the discharge in the neighbourhood of the hole in a cathode offers several features of interest; on some of these, light is thrown by the consideration of the distribution of the lines of force near the hole before the discharge starts.

Let $\mathbf{A}$ and $\mathbf{B}$ be two parallel metallic plates, $B$ having a small hole in it. Let us suppose that $A$ is at a higher potential than $B$, then the negative electricity is not uniformly distributed over $B$ but is very much concentrated near the edge of the hole, the density being infinite at the boundary of the hole if the edge is sharp. The electric force is proportional to the density, so that up to a distance from the hole comparable with the radius of the hole the electric intensity is far in excess of its average value.

Again, this accumulation of electricity is not confined to the part of the plate facing $A$. If the plate $B$ is thin in comparison with the radius of the hole there will, close to the hole, be practically as much electricity on the face of $B$ turned away from $A$ as on the side next to $A$ : in fact the field of force in the neighbourhood of the hole is much as if a fine ring of the same radius as the hole were highly electrified and placed by itself in the field. The appearance of the discharge through the hole when the pressure is not very low illustrates this very well. One of the stages of the discharge is shown in fig. 9 , and it will be seen that this is 
symmetrical on the two sides of the hole. If a strong magnetic field is applied to the neighbourhood of the hole, the lines of force being parallel to the surface of the cathode, some very interesting effects are observed. Those in the

Fig. 9.

Fig. 10.
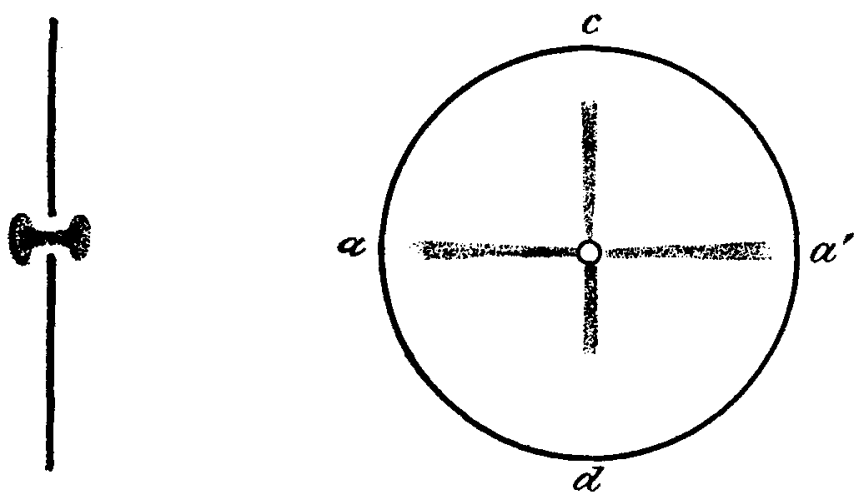

most deve oped form are represented in fig. 10; unless the pressure is within certain limits one or other of these parts may be absent. $a$ and $\alpha^{\prime}$ are two streamers coming from the hole and following the directions of the lines of magnetic force. In this respect they resemble the magneto-cathodic rays of Villard and Righi, but whatever may be the nature of the magneto-cathodic rays in front of the cathode, there can be no doubt, I think, that the rays we are describing consist of slowly moving corpuscles describing spirals of very small radins around the lines of force : for, as the magnetic force is very gradually reduced, we reach the stage when the widening of the radii of these spirals becomes so large that the spiral nature of their path becomes quite obvious. That. they are carriers of negative electricity can easily be shown by sending them into a carefully insulated Faraday cylinder surrounded by another cylinder with a hole in it.

The outer cylinder was put to earth, the inner one was connected with a sensitive galvanometer (the one I used gave a deffexion of $1 \mathrm{~mm}$. at one metre for $3 \times 10^{-10}$ ampere). When one of the streams passed through the hole in the outer cylinder there was a deflexion of 70 or 80 scaledivisions in the direction corresponding to a flow of negative electricity into the Faraday cylinder.

The other two luminous portions $e$ and $d$ are bent at right angles to the direction of the magnetic forces, just 
as they would be if $c$ were a stream of negative and $d$ of easily deflected positive particles. That there were streams of particles of this kind was proved by again directing the streamers into Furaday cylinders connected with a galvanometer. This showed that when the stream $c$ entered the cylinder it carried with it a negative charge, while $d$ carried a positive. $c$ is in most cases much more developed than $d$.

As the pressure is reduced and the dark cathode space gets well developed, the appearances presented by the rays through the hole are very interesting; they are represented in fig. 11.

Fig. 11.

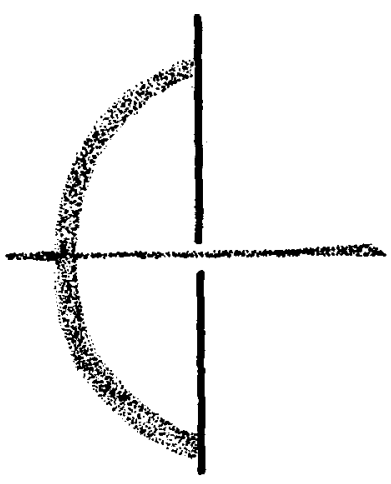

Fig. 12.

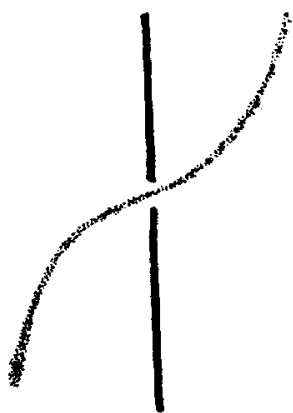

The rays in front of the hole may be traced as a fine welldefined pencil passing through the dark space and often visible for some distance from the negative glow; while it can be traced backwards through the bole for considerable distances, producing, when it strikes the glass walls, the phosphorescence characteristic of cathode rays. Under the action of the magnet the two parts of the pencil are bent in opposite directions, and their appearance shows that they consist of pencils of cathode rays travelling in both directions from the hole (fig. 12), those between the anode and the cathode travelling in the directions of the ordinary cathode rays, whilst those going through the hole travel in the opposite direction, the hole acting as if it sent out cathode rays backwards as well as well as forwards. When the pressure falls below a certain value this pencil disappears, the two portions vanishing simultaneously, and we are left with Canalstrahlen passing through the hole and withont any of the other rays. These other rays can also be made to disappear by the action of a transverse magnetic force, even 
though the pressure is such that they are well developed when the magnetic force is absent.

The value of the magnetic force required to get rid of these rays is very well defined, a change of a few per cent. in the value of this force will entirely alter the appearance of the discharge.

It will be seen that when the Canalstrahlen are accompanied by the other portions of the discharge, there are two sets of positive rays present which suffer very different deflexions in the magnetic field, the Canalstrahlen being very little deflected, while the portion $d$ is very largely deflected. The comparison with the behaviour of the negatively electrified particles shows I think, however, that this difference is due to a difference in the velocity of the particles and not to a change in their mass, for we have seen that there are two streams of negatively electrified particles which are very differently deflected, viz., the rays which constitute the pencil $c$ and those forming the pencils $a, a^{\prime}$ which are bent up into small spirals following the lines of magnetic force. In the case of the two sets of negative rays, we have clearly to do with rays moving with very different velocities; we may regard the more rapidly moving ones as primary rays, the slower ones as secondary rays arising from the ionization of the gas by the primary in weaker parts of the electric field, so that these secondary rays never acquire the velocity of the primary, and are therefore more easily deflected. A similar explanation applies to the two sets of positive rays, the more easily deflected ones arising from the ionization of the gas in the weaker parts of the electric field, where they have no opportunities of acquiring large velocities.

Note on a Method of Measuring the Effective Magnetic Field in the Magnetic Deflexion of the Canalstrallen.

If $O$ represents the mouth of the tube from which the Canalstrahlen emerge, $B$ the screen supposed to be at right angles to the undeflected path of the rays which we take as the axis of $x, \mathrm{H}$ the magnetic force at any point parallel to $z, y$ the deflexion of the rays due to the magnetic field, then

$$
\frac{m d^{2} y}{d t^{2}}=\mathrm{H} e v
$$

if $v$ is the initial velocity of the rays. As the deflexion is 
small this equation is approxinately

$$
\frac{m d^{2} y}{d x^{2}}=\frac{\mathrm{H} e}{v},
$$

so that if $l$ is the distance of the screen from $\mathrm{O}, \mathrm{H}$ the value of the magnetic force at a distance $x$ from $\mathrm{O}$,

$$
\begin{aligned}
y & =\frac{e}{m v} \int_{0}^{l}\left[\int_{0}^{x} \mathrm{H} d x\right] d x \\
& =\frac{e}{m v} \int_{0}^{l}(l-x) \mathrm{H} d x .
\end{aligned}
$$

Hcnce the quantity we have to determine is

let us call it M.

$$
\int_{0}^{l}(l-x) \mathrm{H} d x
$$

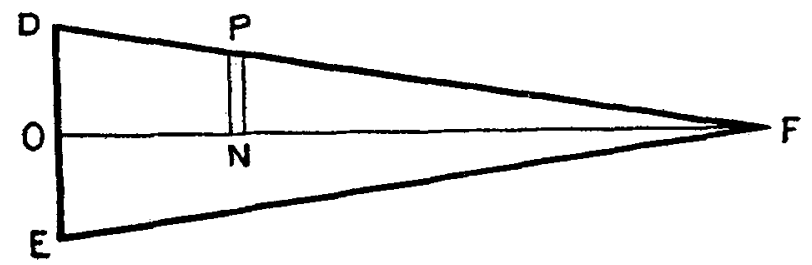

Now suppose we have a coil of triangular section DEF (DF $=E F$ ) placed with its vertex at the screen and its base ED at the hole through which the rays emerge, the plane of the coil being at right angles to the magnetic field. The magnetic induction through the coil

$$
\begin{aligned}
& =\int_{0}^{l} \mathrm{gH} \mathrm{PN}, \quad d x=\frac{\mathrm{DE}}{\mathrm{OF}} \int_{0}^{l} \mathrm{H} \mathrm{FN} d x=\frac{\mathrm{DE}}{\mathrm{OF}} \int_{0}^{l}(l-x) \mathrm{H} d x \\
& =\frac{\mathrm{DE}}{\mathrm{OF}} \mathrm{M} .
\end{aligned}
$$

Hence if we measure the magnetic indnetion through the coil, which can be done in a fow minutes by a Grassot fluxmeter, we can at once determine $M$.

This method takes into account the stray tield and is applicable whether the field is uniform or variable.

I have much pleasure in thanking $\mathrm{Mr}$. Kaye and $\mathrm{Mr}$. Everett for the help they have given me in this investigation. 